\title{
A Spectrum Pooling-based Algorithm in Spectrum Aggregation and Allocation
}

\author{
Feng Peilun ${ }^{1,2}$, Zhang Jianzhao ${ }^{1,2}$, Zhao Hangsheng ${ }^{2}$, Liu Renfu ${ }^{1}$, Li Dali ${ }^{1,2}$ \\ 1. Institute of Communications Engineering, PLA University of Science and Technology, Nanjing, China \\ 2. Nanjing Telecommunication Technology Institute, Nanjing, China \\ feng.peilun@gmail.com
}

\begin{abstract}
Most of existing spectrum allocation algorithms for cognitive radio network are based on continuous spectrum assignment and the user demands are not considered. For this reason, many small spectrum fragments that are smaller than the user demands are generated and these spectrum fragments can't be fully utilized which make a wasteful use of spectrum resources. A new spectrum aggregation and allocation algorithm based on spectrum pooling is proposed in this paper. The discontinuous small spectrum fragments could be aggregated to meet the user demands as much as possible to enhance the spectrum efficiency by using the new algorithm.
\end{abstract}

Keywords- Cognitive radio, Spectrum aggregation, Spectrum allocation, Spectrum pooling

\section{INTRODUCTION}

There is a growing global consensus that the traditional method of spectrum allocation is outdated and inefficient. Substantial spectrum resources are in effect hoard by government, aviation and military users with a little consideration of the opportunity cost to the wider economy. Although the whole framework is changing from "command and control to flexible spectrum management yet its application on the government agencies is limited. In the United States FCC Spectrum Task Force concluded that current spectrum management policies need some reforms. The basic models for the future spectrum management has been recognized as below:

"Command and Control" is the traditional method of frequency spectrum management. The regulator decides the uses and users of the spectrum.

"Exclusive use" where a licensee has exclusive, flexible and transferable rights to use the specific spectrum

"Commons" where unlimited numbers of unlicensed users share the frequencies

Spectrum becomes scarce as wireless technology rapidly develops. But recent research reveals that spectrum is underutilized. Investigating the state of wireless spectrum in the regions (including hot spots of cities), we can find that, lots of spectrum resources is idle in a period of time [1]. According to the differences of times and locations, the usage of licensed spectrum is ranging from $15 \%$ to $85 \%$ [2]. If a secondary user (SU) can access the spectrum opportunity, which has not been occupied by the primary user (PU) in a certain period of time or location, it will greatly improve the spectrum efficiency. The cognitive radio (CR) technology can sense and use the spectrum opportunity, providing an effective way to improve the spectrum efficiency.
Cognitive radio network is different from traditional wireless networks. The availability of spectrum is dynamic, and users need to access to a different spectrum band opportunity. The excellences make the CR-based spectrum allocation method more efficiency than the traditional allocation method. So it is imperative that the spectrum allocation algorithm based on cognitive radio networks should be proposed.

Spectrum pooling is defined as [3]: "some public spectrum which can be rented to users, and gathered in the pool by a certain parameter (bandwidth, quality of service, adjusts requirements, etc.)". It is a resource sharing strategy. The basic idea is that it merges parts of spectrum allocated to different business into a common pool of spectrum and then the channel is divided into a number of sub-channels which is the basic unit in the spectrum allocation. Spectrum pooling sharing strategy is essentially the allocation of limited distribution channels, which aims at maximizing the channel utilization with the fairness of access and minimum of interference considered.

In recent years, most spectrum allocation algorithms are about the continuous spectrum. However in the allocation algorithm of the continuous spectrum, each channel can only be made up of continuous band. It may generate a lot of spectrum fragments which are less than the needs of users, so the spectrum fragments can't be fully utilized and result in a waste of spectrum resources [4]. The non-contiguous spectrum access is possible with the development of Orthogonal Frequency Division Multiplexing (OFDM). In support of that, it can aggregate small fragments of the spectrum as a larger bandwidth and make better use of the spectrum, so as to achieve the purpose of improving spectrum efficiency [5].

The proposed algorithm considers continuity and differences of spectrum opportunity, and facilitates the integrated management of spectrum pooling through the aggregation of various pieces of spectrum. It meets the needs of different users in the allocation to take full advantage of the spectrum, and achieves their needs as much as possible.

\section{SySTEM MODEL}

\section{A. Spectrum Allocation}

The size of spectrum opportunity fragment is random in the wireless environment, which is complex and diversely, causes a lot of inconveniences in the dynamic spectrum management. We design a new method of spectrum allocation based on spectrum aggregation, and it effectively 
resolves the issue that the spectrum fragment is too small to utilize.

Spectrum pooling is a logical pool which store spectrum opportunity fragments in a dynamic spectrum management system. In order to integrate spectrum opportunity resources, this paper presents a spectrum aggregation program which will help the internal management of spectrum, and improve spectrum efficiency, as shown in Figure 1.

We divide the spectrum pooling into several channels, which means the pool can only store several spectrum fragments which are as big as the channels in size. Here we take the design of the base currency principle of RMB in daily life as an example, and set a group of simple but efficient basic channels, so that any band can be divided into a number of basic channels combinations. The basic channel group is made up of seven channels which contain $1 \mathrm{M}, 2 \mathrm{M}$, $5 \mathrm{M}, 10 \mathrm{M}, 20 \mathrm{M}, 50 \mathrm{M}$ and $100 \mathrm{M}$.

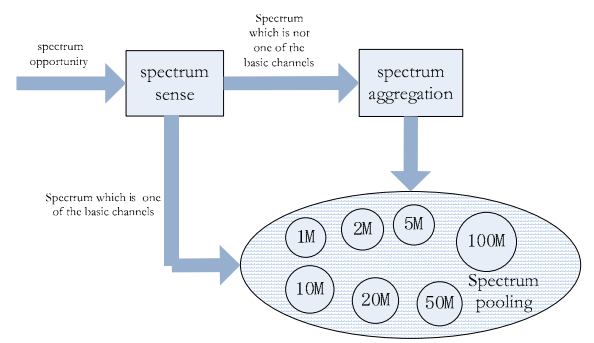

Figure 1. Spectrum aggregation system structure

Spectrum pooling divides spectrum opportunity into two parts, which is in line with the basic channels or not. The first part of the spectrum is directly stored into the pool, and the second part is divided into several basic channels to store in the spectrum pooling management.

Spectrum opportunity fragment is divided into a number of basic channels and a remaining part which is less than $1 \mathrm{M}$. All the remaining parts from every spectrum fragment together can form a new basic channel of the spectrum pooling. The process of spectrum aggregation consists of 4 steps, and relevant algorithms are as follows:

Firstly, spectrum opportunity collected by the spectrum pooling are detected with the basic channels for comparison. If the one has the same specifications as the basis of the channel, the fragment is directly stored into the corresponding basic channel.

Secondly, if the spectrum fragment and the basic channel are not the sane, it needs to be split. According to the order of basic channel from large to small in sequence, a larger basic channel can be decomposed from the spectrum fragment, and stored into the spectrum pooling.

The third step is re-detecting the rest of the spectrum opportunity, and repeating the previous steps.

Finally, if the fragment of the last remaining spectrum opportunity is less than $1 \mathrm{M}$, it should be stored into fixed spectrum storage for a while. After several cycles, the storage achieves a certain number of spectrum opportunities, which can be stored as those steps listing in front.
Algorithm flow chart has been shown in Figure 2.

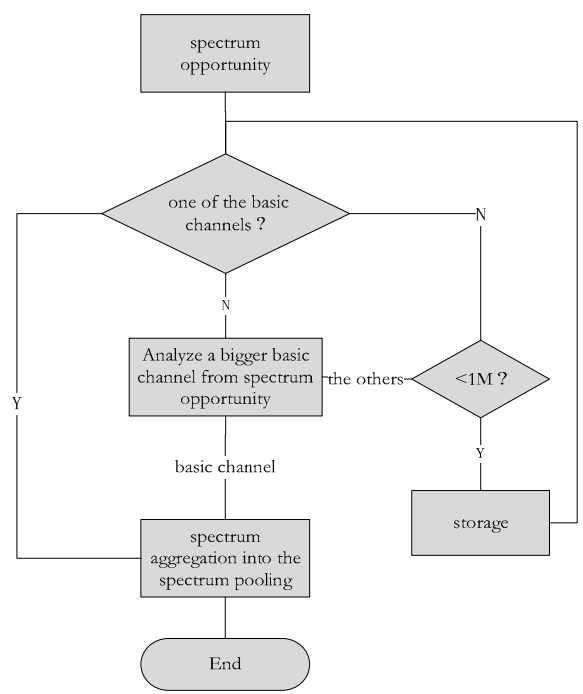

Figure 2. The algorithmic flow of spectrum aggregation

As the wireless environment is constantly updating, the collected spectrum opportunity is stored uniformly and orderly in the pool through spectrum aggregation, which facilitate the management of the pool. Then they are ready to be reserved by cognitive users at any moment. In this paper we assume no cognitive users need spectrum from the pool in the short interval of the spectrum aggregate.

\section{B. Spectrum Allocation}

Spectrum allocation discussed in this article is based on the spectrum pooling above and cognitive users select spectrum from the spectrum pooling to fulfill their requirements. The mechanism of spectrum allocation is similar as the principle of aggregation, which is the combination of the basic channels of the spectrum pooling to form the required spectrum for the cognitive users. Spectrum allocation process is the reverse of the aggregation. We design the schematic diagram through referring to the chart of the spectrum aggregation, which is shown in Figure 3.

According to the needs for spectrum of cognitive users, the method selects a few channels to satisfy their satisfaction. There are several programs when one requirement is achieved. For example, a commodity is valued at 70 Yuan. So it can be composed of a 50 Yuan and a 20 Yuan, a composition of a 50 Yuan and two 10 Yuan's, and one by the composed of three 20 Yuan's and one 10yuan. Of course, there will be many different combinations. However, it is evident that the first is the most concise and efficient. Therefore, we select the most concise and efficient method of combination in the spectrum allocation, namely, choosing larger channels as much as possible to meet the needs of cognitive users. 


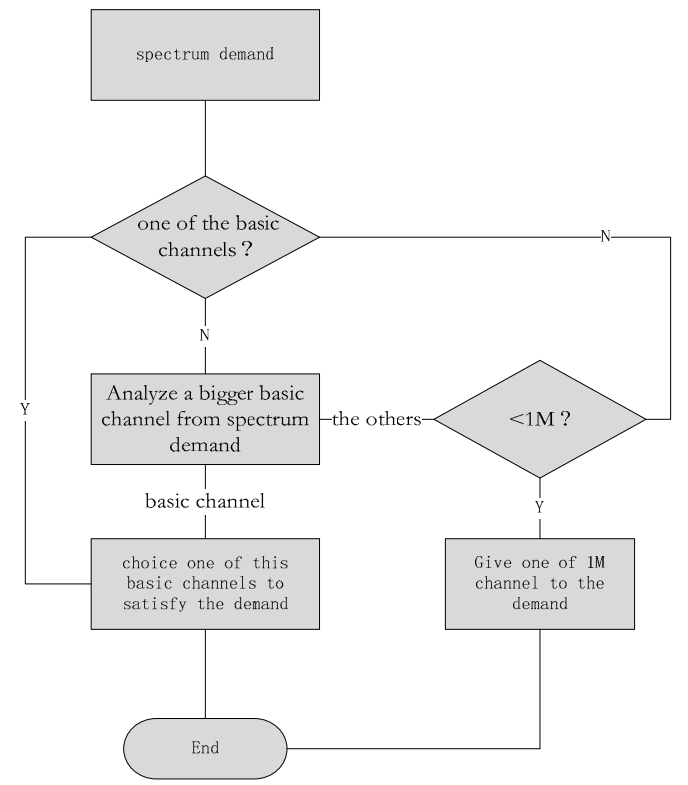

Figure 3. The algorithmic flow of spectrum allocation

When spectrum needs of cognitive users are not just based entirely on the basic channels in the spectrum pooling, a basic channel in the pool has to provide services to meet the requirement of a spectrum fragment which less than $1 \mathrm{M}$ of the cognitive users. Thus the utilization of this $1 \mathrm{M}$ basic channel is reduced. But the utilization of the other basic channels which are be used by the same CR simultaneously has reached $100 \%$. So the overall utilization rate maintains at a high level, while meeting the user's requirements about spectrum to ensure quality of service of spectrum pooling. So a waste of a small part of spectrum resources is acceptable and can be ignored.

\section{SimUlATION}

In order to validate the performance of the proposed allocation method which bases on the aggregation of spectrum, we use MATLAB to build a simulation platform for dynamic spectrum allocation. To highlight the problem to be explained, we have not consider the factors unnecessary, and assume that all the standard conditions of the spectrum fragments are the same except the size.

The proposal algorithm is compared with the greedy algorithm, randomized algorithm and collaboration algorithm which are proposed in the article [6], and the evaluation parameter are spectrum utilization efficiency, throughput and time delay of each algorithm.

The comparison of four proposed algorithms is shown in figure 4 - figure 6 . We can see that, as the secondary users' increases, the advantages of the proposed algorithm is more evident. This is because in times when the user less, the free spectrum resources more, resulting in lower spectrum efficiency overall. More resources are occupied by SUs as the number of SUs gradually increased, and small spectrum fragments generated from continuous spectrum increased too. Thus the proposed algorithm, which ravels out allocation of the remaining small fragments, improves the spectrum utilization compared to several other algorithms significantly. As the throughput is improved also, the time delay of the proposed algorithm is much more better than the other three algorithms.

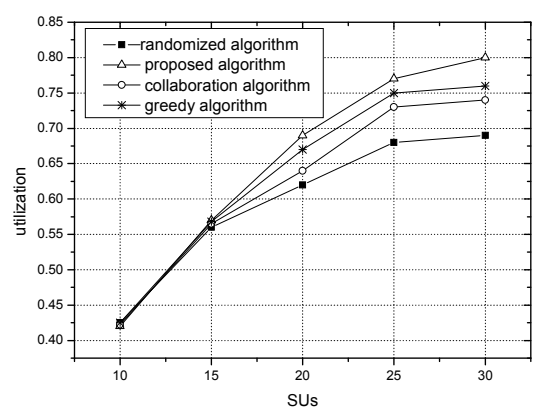

Figure 4. Utilization for the four algorithms

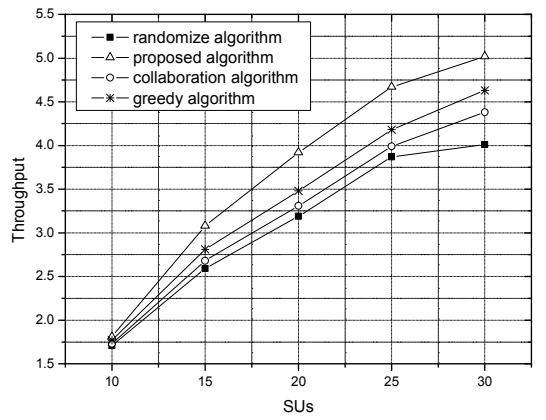

Figure 5. Throughput comparison for the four algorithms

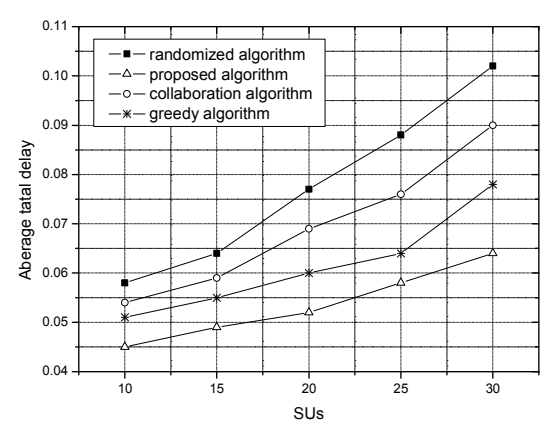

Figure 6. Time delay comparison for the four algorithms

\section{CONCLUSION}

Improving spectrum efficiency is the main problem needed to solve in dynamic spectrum allocation and this article proposed a method of aggregation and allocation of spectrum. It is an effective solution to the non-continuous 
spectrum resources utilization, and improves the spectrum utilization rate evidently.

An efficient algorithm for spectrum aggregation was proposed. Our simulation results show a significant performance improvement with dynamic clustering scheme. Compared to the traditional algorithms that do not consider small spectrum fragments to unused spectrum, our algorithm has the advantages of requiring less time delay for managing spectrum and adapting to the radio environment. The approach presented permits all users to improve the throughput and utilization. Because all users need the spectrum, the algorithm allows users to use the spectrum as possible as perfect. The algorithm is robust against any router failing. Coordination traffic congestion that resulted from coordination messages is reduced by organizing users into clusters. In future, we wish that channel allocation for SUs in cognitive network will be considered in future work.

\section{ACKNOWLEDGMENT}

This paper is funded by the National Natural Science Foundation (61072077)

\section{REFERENCES}

[1] Simon Haykin. Cognitive radio: brain-empowered wireless communications. IEEE Journal on Selected Areas in Communications, 2005, 23(2): 201 220

[2] Akyildiz I F, Won-Yeol Lee, Vuran M C, et al. Next generation/ dynamic spectrum access/ cognitive radio wireless networks: a survey. Computer Network, 2006, 50(13): 2127 2159

[3] Fatih Capar, Ihan Matoyo, Timo Weiss, and Friedrich Jondral. Comparison of bandwidth utilization for controlled and uncontrolled channel assignment in a spectrum pooling system. In Proceedings of the IEEE 55th Vehicular Technology Conference VTC Spring 2002, pages 1069-1073, Birmingham (AL), 2002

[4] Chen Dawei, Zhang Qian, Jia Weijia. Aggregation aware spectrum assignment in cognitive ad-hoc networks. In: 2008 3rd International Conference on Cognitive Radio Oriented Wireless Networks and Communications, Singapore, May 2008

[5] Dr Anil Shukla, Brian Willamson, John Bums, et al. A study for the provision of aggregation of frequency to provide wider bandwidth services. A Final Report of QinetiQ. QINETIQ/06/01773, August 2006

[6] Liu Shuai, Liu Yu-tao, Tan Xue-zhi, et al. Cooperated Bidding Algorithm for Spectrum Allocation Based on Maximal Independent Set. Science. Technology. Engineering, 2009,9(17) 